\title{
Reporting systems in healthcare from a case-by-case experience to a general framework: an example in anaesthesia
}

\author{
A. S. Nyssen*, S. Aunac ${ }^{\dagger}$, M. E. Faymonville ${ }^{\Uparrow}$, I. Lutte ${ }^{\ddagger}$ \\ ${ }^{*}$ University of Liège, Department of Work Psychology, Liege; ${ }^{\dagger}$ Cliniques Universitaires St Luc, Department of \\ Anaesthesiology, Brussels; "University Hospital of Liège, Department of Anaesthesia and \\ Intensive Care Medicine, Liege; ${ }^{\ddagger} U L B$, Department of Law, Brussels, Belgium
}

\begin{abstract}
Summary
Reporting systems are becoming more widespread in healthcare. Since they may become mandatory under the pressure of insurance companies and administrative organizations, it is important to begin to go beyond a case-by-case approach and to move to a system where there is a general reflection on the best conditions of development and setting up of such systems in medicine. In this paper, we review existing reporting systems, break down their components, examine how they are constructed and propose some ideas on how to articulate them in a dynamic process in order to improve the validity of the tool as mediator of safety, quality and well-being at work.
\end{abstract}

Keywords: ANAESTHESIA, REPORTING SYSTEMS; SAFETY MANAGEMENT; RISK MANAGEMENT; QUALITY ASSURANCE, HEALTHCARE.

Following their development in industrial and aviation areas, accident reporting systems are becoming more widespread in healthcare. The intention behind their implementation is to systematically collate and analyse the risks associated with medical activities in order to propose remedial and preventative actions. Several factors can explain the expansion of such systems in the medical sector. First, the progress of technology in general together with the development of increased technology in medicine itself have shed light on the role played by human errors in medical accidents. Reporting systems therefore first focused on the identification of patient injuries attributable to human errors. Second, the relationships between doctors and patients have changed and the public has been calling for clearer information

Correspondence to: Anne-Sophie Nyssen, Department of Work Psychology, University of Liège, Bat. B-32 FAPSE, 4000 Liege, Belgium. E-mail: asnyssen@ulg.ac.be; Tel: +3243664666; Fax: +3243662944

Accepted for publication June 2004 EJA 1624 concerning the risks associated with medicine. Third, litigation for medical negligence has increased to such an extent in many English-speaking countries that insurance companies, administration organizations and state health ministries have pressed for the development of strategies that can proactively prevent injuries. Professional organizations also have played a role in the development of reporting systems. Fearing medico-legal consequences, doctors and hospital staff have started to become aware of the need to change the myth of an error-free system in medicine and see in reporting systems one means to change culture and attitude towards human errors.

In a recent report produced by the Quality of Health Care in America project [1], different existing reporting systems in medicine were reviewed. They varied according to a number of features. Some systems were mandatory by internal or external structures, whereas others were voluntary and confidential. Some systems focused on adverse patient injuries while others were extended to any event which could have, or did harm, anyone in the hospital, including 
staff and visitors. Some systems aggregate data, others propose remedial actions as well as feedback to the reporters. Besides this variety, it is imperative to go beyond a case-by-case experience and to start a general reflection on the best conditions of development and setting up of such systems in healthcare. The Belgian Federal Government Prime Minister's Offices provide the opportunity for this work to be co-ordinated. A multi-disciplinary research network including legal specialists, doctors, psychologists and a medical administrator has been set up to develop proposals for ongoing reporting and analysis of failures and accidents in the context of Belgian healthcare. In this review, we present the first reflections of this network. We break down reporting system components, see how they are elaborated and propose some ideas on how to combine them in a dynamic process in order to improve the validity of the tool.

\section{Reporting system process}

Figure 1 describes the many different functional steps that must be fulfilled in order to drive any reporting system: development of the reporting interface, data collection, data analysis, recommendations, implementation and evaluation.

\section{Accident/incident/event}

A reporting system, by definition, is a systematic gathering of information about accidents. The first difficulty is that of the definition of an accident. We $\begin{array}{lll}\text { Approach } & \text { Tools } & \text { Steps }\end{array}$

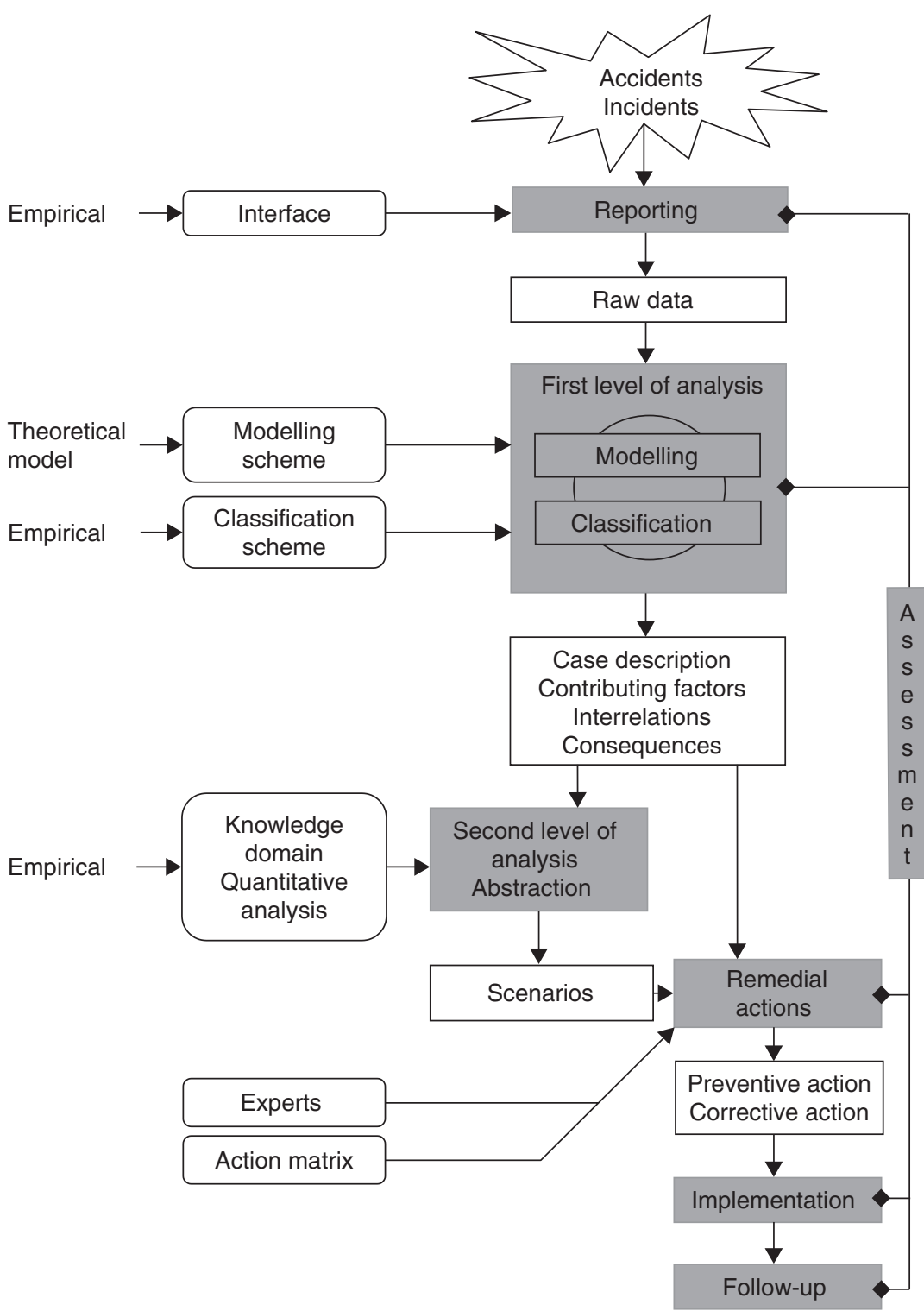

Figure 1.

Reporting system components. Grey rectangles represent reporting system steps, white rectangles represent the products of each step. 
have found at least three different meanings to the term accident and error within the working group: one psychological, one medical and one legal. Ambiguity can thus arise about what the system aims to collect as information. Traditionally, reporting systems have focused on accidents due to human errors. In recent years they have extended their scope to critical events that either could have or did harm someone. This has the advantage of favouring the collection concerning any type of problem situation, including those that ordinarily come up at work but which do not bring on harmful consequences because they have been treated in time (this incident category is also referred as a 'near miss').

There are several biases in what is reported. A study carried out in an Australian hospital [2] shows that in $80 \%$ of cases, the accident reports are generated by nurses and they are generally limited to the problems which are relevant to nurses, such as medication problems. Due to this bias, the reports cannot be used as a statistical basis for estimating accident probability.

\section{Capturing the event}

Reporting is achieved by means of an interface. Three types of interface can be used: questionnaire (or reporting form), interview and automatic data record. The most common interface used is the questionnaire. In certain hospitals, there are more than eight separate cards to fill out. The tendency is thus to avoid the reporting procedure and to report only the accidents that lead to serious harm to the patient. The questionnaire is usually presented in the form of a multi-axial framework comprised of questions and scales measuring several variables concerning the event. The question format can be either closed (multiple choice) or open-ended (narrative section). The content of the questionnaire is predetermined and, at the same time, reflects the model of causality that the designer has of the event. Thus, in identifying questionnaire content, it is best to first list the risks on which information is to be collected. This can be done on empirical observations or on a theoretical basis. In recent years, a consensus has appeared among researchers to consider an accident as a symptom of a human-system mismatch resulting from multiple causes rather than of one unique cause (technical or human). This means that most of the time, there are questions concerning multiple data including technical, organizational and individual variables. Questionnaires allow for the collection of highly structured information about accidents, but, at the same time, the predetermined structure involves a loss of information. The use of interviews can help to reduce this bias. Nevertheless, interviews come with a high cost in time and resources, and experience is required to collect valuable information.

Recently, another type of interface has been used to collect information about accidents. Sanborn and colleagues [3] have used a computerized anaesthesia information management system to scan case records electronically for deviations from specific limits for physiological variables. Such interfaces are extremely valuable in measuring frequencies and sequences of patient data, but the sheer size of the automated recorded information is difficult to use with a view to develop or verify causal analysis between the related accident variables.

\section{Data analysis}

Reporting systems include a method to analyse data and involve a classification scheme. In many reporting systems, the classification scheme is built empirically on the basis of the reported data and is domain specific. As example, the Generic Occurrence Classification developed by Runciman and colleagues [4] is specifically for the medical field. It was constructed by an iterative process in which 'natural categories' were identified from over 2000 incidents and 800 adverse events, and placed in a hierarchical structure [5]. In other reporting systems, the classification is derived from psychological models and makes a distinction, for example, between slips and mistakes [6] or between active and latent failures.

Even if there is now a consensus among experts to define an accident as a system failure, analyses illustrating the multi-causal aspect of an accident are still rare. In most studies, the related accident variables (technical, individual and organizational) are considered in isolation. There are, in fact, few techniques that can be used to describe the relationships between these variables. The causal tree diagram has been used by several researchers to visualize graphically the multiple conditions leading to an accident $[7,8]$.

In recent years, several investigators have looked farther into the idea of the multi-causality of accidents and have questioned the extent to which a set of factors extracted from analyses of accidents are important influences on accidents compared to events or histories $[9,10]$. In other words, does thinking about accident characteristics tell us about why failures occur at work? For example, in order to understand the significance of someone's behaviour at work, knowing about the associated factors is certainly important, but what may be much more relevant is the 'history' of the person in the context of how the event came over time. The idea is to provide a description of the environment that was directly relevant to the conducting of the behaviour. From this perspective, it is the constant processes of interaction 
between the person and the environment that is important, rather than particular characteristics of the person or the environment at any particular time. The major challenge is to distil the cases into a smaller number of scenarios containing the critical issues concerning accident analyses. Cook and colleagues [10] have used the technique to collect a series of cases in anaesthesia illustrating some generic cognitive difficulties. One immediate implication of this approach is that the initial assessment using formal categories and showing human error as the major cause of accidents should be supplemented by techniques that can assess behaviour in context.

\section{Construction of remedial and preventative actions}

The next step of a reporting system consists of the proposal of remedial and preventative actions. Different approaches can be used to construct them. The 'Classification/Action Matrix' developed by van Vuuren [6] determines a priori the most effective actions according to the category of failure. In the Edinburgh intensive care unit's reporting system [11], the remedial actions are constructed in a collaborative process by a group of experts based on the results of the data analyses. Since the database can be very large and diverse, there is a need for setting priorities. Traditionally, responses in healthcare have been set on rates of mortality and severe injuries. But a broader approach is required as much of the cost of iatrogenic injury is created by high-frequency and relatively lowseverity events. The responses can be very diverse; they may imply technological, ergonomic, organizational or training measures. They may be set at different levels: individual, departmental, systematic, national and international. These measures are not exclusive.

It is clear that reporting systems are based on the confidence established between the reporter and the analysis made of the reported data. But it is now recognized that feedback plays an important role in the long-term adherence of the staff to the system.

\section{Implementation and follow-up}

The main critical step in a reporting system is certainly the implementation and follow-up phases. Indeed, reporting systems have been implemented in many hospitals, but then abandoned. There are several reasons for this: the length of the questionnaire, the lack of user involvement, the fear of blame, the lack of feedback and the running costs. Some recommendations have been made in the Quality of Health Care in America project as well as in the Australian report made by Runciman [12] and colleagues. In our view, successful implementation involves establishment of the framework well ahead of time. This implies that the goal of the reporting system is clearly established and understood by the different partners.

Four frames must be considered: medical, technical, organizational and legal. At the level of the medical frame, the collaboration and the involvement of the domain experts is indispensable to the collection and analysis of the data. At the level of the technical frame, the techniques of analysis (classification and accident formalization) together with their tools must be chosen according to the goal pursued. It is necessary to determine the form in which (computerized, verbal or written) the tools and the data would be accessible to the various users (the reporters and the analysts). The technical frame must be sufficiently flexible in order to be able to be adapted to the various possible uses (safety improvement, learning or research). At the organizational level, designers should make sure that the reporting system project is positioned, from the beginning, within a culture of quality and patient safety improvement rather than a 'blame culture'. A large part of the effectiveness of the system depends on this. Several structural points must also be negotiated with the administration staff such as who will be responsible for the system, who will have access to the system and how that should be done, the degree to which information will be confidential or anonymous and what kind of feedback and dissemination of information will be given. Such questions should have an answer before the implementation of the system in order to favour its long-term usability within the system. The last frame, the legal frame, is certainly as delicate as the organizational frame. At that level, the data gathered, along with the reporters and the managers of the systems must be protected. The protection can be developed along two axes: a legal axis and a pragmatic axis. On the legal axis, there is already a series of protections that are registered in the laws (e.g. professional secrecy) and that can, possibly, be taken into account. However, we can also imagine that the legislature could grant some kind of privileged status to reporting systems that would protect them from legal investigations. On the pragmatic axis, it is possible to ensure protection through several techniques: a confidential processing of information collected, a transmission of information in an anonymous way and a de-identification of the reports once completed.

\section{Assessment}

Up until now, reporting systems which include an assessment phase have been rare. Although the principal goal of the reporting systems is to create a 
database and identify the risk of accidents in order to reduce them, they affect culture and also produce changes in the people who are working within the system. For this reason, it is important to assess the tool in context and evaluate what short- and longterm effects they have on the general work situation. The scope of the reporting can be evaluated, the classification and analysis schemes can be reviewed according to their practicability. The effectiveness of the remedial and preventive actions can be studied in short- and long-term perspectives.

\section{Application of the Incident Reporting System in Belgium}

In 1999, the Belgian Federal Government Prime Minister's Offices funded our research group to conduct an incident monitoring pilot project as a method to improve well-being at work. Theoretical foundations for this can be found in the scientific literature on 'risks in high-performance work systems' showing the role played by organizational factors. Many experts in human factors encourage a culture shift which acknowledges that providers do not fail alone. Organizations and systems have vulnerabilities, as do individuals, and the ingredients of many accidents are present long before a specific incident occurs. These latent features, combined with an inexperienced or fatigued caregiver may produce an equipment failure or a medical mishap. We think such a mishap represent systems failure. Today, more than ever, healthcare workers face unprecedented demands for production, safety, efficiency and value. Recent trends to reduce the financial losses in the healthcare system have exacerbated pressures on workers leading in a number of major medical corporations to strike actions. In a previous study [13], we showed that $40.4 \%$ of the French-speaking anaesthetists working in three Belgian Universities were suffering from high-emotional exhaustion (burnout) the highest rate concerning young residents under $30 \mathrm{yr}$ old. The 'work organization', more specifically the lack of control over work, time planning and risks, the lack of supervision and communication within the team, especially with the surgeons were perceived as the major sources of stress. The implication of doctors' responsibility in accident investigation only increases these inherent stressful working conditions. Together, they can lead to impaired performance and health $[14,15]$.

One of the challenges of this project is to link quality, security and well-being at work. Our project is the first of its kind in Belgium. We were aware that such an information system could, if no precautions were taken, possibly compromise workers targeted by the declaration. That is why the working group which prepared the project included legal specialists, doctors and psychologists in order to resolve the ethical and legal problems of confidentiality and responsibility and to facilitate the change of culture within the working situation.

We chose to concentrate on one medical specialty frequently confronted with medical lawsuits, namely anaesthesia and to apply the process of reporting and analysing incidents and accidents in two different anaesthesia departments of university hospitals of Belgium. The experiment has been progressively widened to other high-risk hospital sectors such as intensive care and emergency medicine using the same specialized reporting form before being extended to other hospitals with a view to organizing a federal structure.

A wide variety of reporting systems and relevant theoretical information has been consulted in order to develop the reporting form. The actual form ${ }^{1}$ is quite detailed and holds fields required for the identification of the event and other fields necessary for the administration of the form. It has two components. Part A contains a free general description (narrative part) of the incident in which the reporter is asked to write a description of what happened in their own words. Part B asks for a detailed description of the incident using a systematic approach. The form is prefaced by an invitation to complete the form, asking the person to report any incident which did, or could, affect the safety of a patient whether or not it may be preventable. The identity of the reporter and the patient do not appear on the report and the exact time, date or location are not requested.

The narrative description section (Part A) aims to collect a chronological and logical chain of the events surrounding the incident in order to obtain a picture of the history and development of the incident: "In your own words describe the incident. Include details about any factors which you believe may have contributed to, minimized or prevented the incident. Do you think this incident was preventable?" Key events will be coded by the analyst into the database. The objective is to facilitate analyses and identify risk that might lead to other incidents.

The system-wide event description (Part B) is designed to elicit the salient features of an incident placed in the context of the whole. The classification of the incident begins with the failure itself in terms of the domain. The idea is based on the concept of classifying each incident into one or more natural categories with each incident being linked to its contributing factors, preventive factors and factors

\footnotetext{
${ }^{1}$ Original copies of the questionnaire can be obtained directly from the corresponding author.
} 
Table 1. What happened?

\begin{tabular}{ll} 
Circuit problem & Equipment involved \\
Dilution of gases & Airway, mouth prop, gag \\
Disconnection & Airway pack \\
Leak & Diathermy, cautery, shock \\
Misconnection & Equipment malfunction \\
Overpressure & Infusion apparatus \\
Rebreathing & Intubation aid \\
Other circuitry incident & Laryngoscope \\
Circuit involved & Monitoring equipment \\
Absorber & Nasogastric tube \\
Common gas outlet & Mask \\
Endotracheal tube & Needle or cannula \\
Flowmeter & Strapping or tape \\
Gas supply & Syringe \\
Humidifier & Suction apparatus \\
Oxygen bypass & Table or trolley \\
Circuit valve & Patient support \\
Pressure relief valve & Other equipment \\
Scavenging system & \\
Connecting tubing & \\
Vaporizer & \\
Ventilator & \\
Other & \\
\hline
\end{tabular}

Pharmacological incident
Allergy
Contamination
Inappropriate drug
Interaction
Overdose
Side-effect
Wrong drug
Underdose
Other drug incident
Airway incident
Difficult intubation
Endobronchial intubation
Extubation
Failed intubation
Non-ventilation
Obstruction (including vomit or spasm)
Oesophageal intubation
Trauma
Other airway incident minimizing outcome (detection factors). Those factors can be broken down into four large subclasses: equipment factors, individual factors, team factors and organizational factors. These are summarized in Tables $1-5$. The questionnaire is built from these questions with response boxes inserted in appropriate places or instructions to circle the correct responses. In addition, we have integrated into the form the search for information concerning how the worker has coped with the event and its impact on his/her health (Table 5). We, in addition, include at the end an assessment of the questionnaire itself asking the person completing it for comments on clarity, structure and ease of its completion. We anticipate and hope that it will be possible to document the link between safety, quality and well-being at work in order to improve social or medical support.

In $1 \mathrm{yr}$, more than 200 events from the two anaesthesia departments in our hospital have been reported using the same reporting form and coded in a common database. It is not the purpose of this review to present the results of the data analysis. It is useful, however, to report that following our proposed methodology, direct preventive and corrective responses to specific events have already been recommended and implemented in the two hospitals. This has improved the frequency of reporting. Among these, an emergency call procedure has been introduced in order to support junior staff confronted with dynamic situations, an airway management skills programme is in place to respond to the frequently reported difficult intubations, a simulator training programme has been organized on communication and crisis management and individual psychological support is available to provide special support after accidents.

Our experience has clearly demonstrated that given a suitable structure and approach, the Incident Reporting System will be able to provide the data required to evaluate risks in the health sector with a view to improving the work. In our experience, the workers have been closely involved in the project activities. This constitutes a solid basis for successful change at work. In addition, the involvement of legal specialists and psychologists as partners in the work group reinforce the legitimacy of the project.

\section{Conclusion}

These first reflections on reporting systems have, as their main goal, the ability to attract attention to one error prevention tool that is becoming more widespread in healthcare and which is experienced on a case-by-case basis. From this arises the necessity of developing a design framework allowing for a more systematic appraisal of the tool. Our review illustrates the need for an interdisciplinary team including domain experts, legal experts, analysts and psychologists, for successful designing and implementing. The need for an international benchmarking 
Table 2. Why did the incident occur?

\begin{tabular}{|c|c|c|}
\hline Contributory factors & Minimizing factors & Suggested corrective strategies \\
\hline \multicolumn{3}{|l|}{ Equipment } \\
\hline Lack of facilities & Monitor detection & Additional equipment \\
\hline Lack of monitor & Identify which monitor & Additional monitor \\
\hline Monitor problem & Early detection & Equipment checking procedure \\
\hline Design problem & Assistance in diagnosis & Better equipment design \\
\hline Control problem & Adequate security measures & Better equipment maintenance \\
\hline Unfamiliar equipment & Re-check equipment & \\
\hline \multicolumn{3}{|l|}{ Other equipment problem } \\
\hline \multicolumn{3}{|l|}{ Equipment not available } \\
\hline \multicolumn{3}{|l|}{ Drug label error } \\
\hline \multicolumn{3}{|l|}{ Individual } \\
\hline Distraction & Healthy patient & Additional training \\
\hline Error of judgment & Knowledge of the patient & Fatigue alleviation routine \\
\hline Error of diagnosis & Re-check & \\
\hline Error of confusion & Awareness of danger & \\
\hline Fault of technique & Prior experience & \\
\hline \multicolumn{3}{|l|}{ Error of procedure } \\
\hline \multicolumn{3}{|l|}{ Error to check } \\
\hline \multicolumn{3}{|l|}{ Overconfidence } \\
\hline \multicolumn{3}{|l|}{ Inattention } \\
\hline \multicolumn{3}{|l|}{ Fatigue } \\
\hline \multicolumn{3}{|l|}{ Haste } \\
\hline \multicolumn{3}{|l|}{ Pressure to proceed } \\
\hline \multicolumn{3}{|l|}{ Inexperience } \\
\hline \multicolumn{3}{|l|}{ Inadequate training } \\
\hline \multicolumn{3}{|l|}{ Inadequate knowledge } \\
\hline \multicolumn{3}{|l|}{$\begin{array}{l}\text { Inadequate/incorrect preoperative } \\
\text { patient assessment }\end{array}$} \\
\hline \multirow{2}{*}{\multicolumn{3}{|c|}{$\begin{array}{l}\text { Inadequate/incorrect preoperative } \\
\text { patient preparation }\end{array}$}} \\
\hline & & \\
\hline \multicolumn{3}{|l|}{ Sick patient } \\
\hline \multicolumn{3}{|l|}{ Other stress } \\
\hline \multicolumn{3}{|l|}{ Team } \\
\hline Communication problem & Good communication & Improved communication \\
\hline Co-ordination problem & Relief anaesthetist & Improved staff \\
\hline Inadequate supervision & Staff change & \\
\hline Relief anaesthetist & Skilled assistance & \\
\hline \multicolumn{3}{|l|}{$\begin{array}{l}\text { Relief anaesthetist } \\
\text { Staff change }\end{array}$} \\
\hline \multicolumn{3}{|l|}{ Sick anaesthetist } \\
\hline Surgical team contribution & & \\
\hline Organization & & \\
\hline Information transmission problem & Good supervision & Improved environment \\
\hline between services & Procedures & Improved supervision \\
\hline Planning problem & Adequate resources & More manpower \\
\hline Lack of support from & Other factor & Quality insurance activities \\
\hline Surgeon & & Specific protocol development \\
\hline Team & & \\
\hline Hospital & & \\
\hline Family & & \\
\hline Due to the emergency nature & & \\
\hline Other factor & & \\
\hline
\end{tabular}

to align some of the objectives and methodologies already in use in the existing systems is also evident. Furthermore, it is important to go beyond the simple collection of information and to start an in-depth reflection on the methodology of analysis and on the way responses are made to reported incidents. It is clear that computerization of the work sector represents an ideal means for collecting data about accidents, but in general these databases are hardly ever used within the system. At the same time, it is 
Table 3. Anaesthesia, procedure and time.

\begin{tabular}{|c|c|c|}
\hline Procedure & Monitors in use & When did it occur? \\
\hline Cardiothoracic & Airway pressure gauge & Time of day \\
\hline Cardioversion & Airway temperature & Month \\
\hline Dental & Auto-disconnect alarm & \\
\hline Diagnostic including imaging/biopsy & Manual disconnect alarm & At what phase? \\
\hline Electroconvulsive therapy & Capnograph & Pre-induction \\
\hline Endoscopy & Cardiac output & Induction \\
\hline Ear, nose and throat & Central venous pressure & Maintenance \\
\hline General surgical & Doppler praecordial & Emergence \\
\hline Gynaecological & Endotracheal cuff pressure & Recovery \\
\hline Haematological & Electrocardiograph & Post-recovery \\
\hline Major multi-disciplinary & Electroencephalograph & Location \\
\hline Maxillo-facial & Intra-arterial pressure & Casualty or emergency room \\
\hline Neurosurgical & Intracranial pressure & Day-surgery \\
\hline Obstetric & Mass spectrometer & Delivery room \\
\hline Oncology & Nitrous oxide analyser & Dental room \\
\hline Ophthalmology & Other gas analyser & General ward \\
\hline Orthopaedic & Pulse oximeter & Imaging \\
\hline Plastic or reconstructive & Oxygen analyser & In transit \\
\hline Radiotherapy & Plethysmograph & Induction room \\
\hline Urology & Peripheral nerve stimulator & Intensive care \\
\hline Vascular & Pulmonary arterial or wedge pressure & Operating room \\
\hline Other procedure & Sphygmomanometer & Other in hospital \\
\hline Elective Yes/No & $\begin{array}{l}\text { Auto } \\
\text { Manual }\end{array}$ & $\begin{array}{l}\text { Other outside hospital } \\
\text { Recovery area }\end{array}$ \\
\hline General anaesthesia & Spirometer & \\
\hline Infiltration & Stethoscope & \\
\hline Regional or nerve block & Praecordial & \\
\hline Sedation & Oesophageal & \\
\hline No anaesthesia & Patient temperature & \\
\hline $\begin{array}{l}\text { Type of ventilation } \\
\text { Spontaneous } \\
\text { Controlled }\end{array}$ & Other monitor & \\
\hline
\end{tabular}

Table 4. Persons involved.

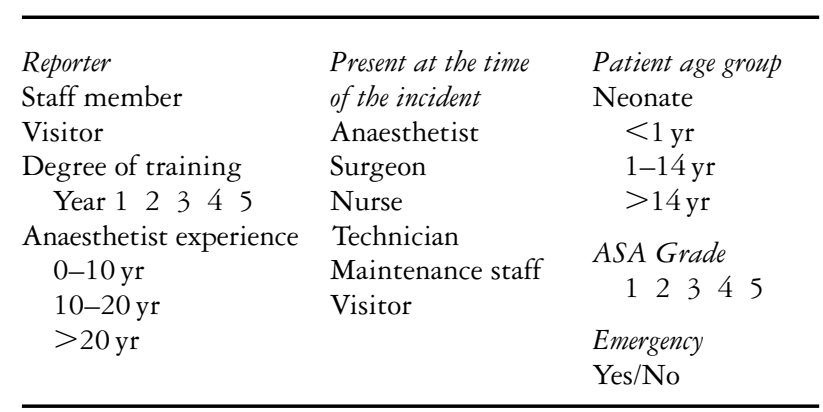

mostly the practitioners that are interested in reporting systems. Moreover, reporting and analytical approaches must be simple to use and produce results that make sense for them. In this paper, we put forth the idea of scenarios, characterized by some specific combinations between the different factors at the origin of accidents: contextual, organizational and cognitive factors. The unit of analysis is semantic which, in turn, has certainly more significance for practitioners. Indeed, we believe that the concept of scenarios could provide a mechanism for abstracting common generic patterns of complex system breakdowns. However, future studies should investigate the predictive validity of this analytical approach. Another important direction for analysis of reported data is the study of accident and error detection and recovering processes. Although the consequences of an accident depend largely upon the amount of time and activities that intervene between the accident and its discovery, there have been very few studies on that theme. Reporting systems could serve to investigate this research issue, one with considerable theoretical and practical interest. Finally, it is worth to noting that, although reporting systems constitute a valuable tool for a better understanding of the genesis of accidents, under no circumstances are they a substitute for field observations and in-depth knowledge of the domain in order to make sense of the reported data. 
Table 5. Detection, evolution and aftermath.

\begin{tabular}{|c|c|c|}
\hline Detected by? & Duration of incident & After the incident \\
\hline Self & Transitory $(<5 \mathrm{~min})$ & Was there a feeling of: \\
\hline Other person (specify) & Prolonged $(>5 \mathrm{~min})$ & Anger at the equipment \\
\hline None & & Anger at myself \\
\hline How? & Immediate effects & Anger at the team \\
\hline Alarm & Cardiac arrest & Anger at the organization \\
\hline Alarm & Major physiological change & Culpability \\
\hline Ask/call & Minor physiological change & An unanswered question \\
\hline Checking procedure & Physical injury & Did you notice: \\
\hline $\begin{array}{l}\text { Problem suspicion } \\
\text { Based on expectancy }\end{array}$ & Nil & Lack of sleep \\
\hline $\begin{array}{l}\text { Based on expectancy } \\
\text { Based on knowledge }\end{array}$ & & Loss of appetite \\
\hline By chance & Long-term effects & Difficulty of attention \\
\hline Other (specify) & Awareness & Obsessive thoughts \\
\hline Utner (speciry) & Death & Loss of pleasure \\
\hline When? & Major morbidity & Fear \\
\hline Immediately & Minor morbidity & Other emotion \\
\hline Delayed & Prolonged stay & \\
\hline By how long? & Unplanned high-dependency stay & \\
\hline Never & Nil & \\
\hline
\end{tabular}

\section{Acknowledgements}

This work was supported by the Belgian Federal Government, Prime Minister's Offices, OSTC Program Phase II (Worker protection in the area of health). We would also thank Dimitri Mouffet for his contribution.

\section{References}

1. Kohn LT, Corrigan JM, Donaldson MS, eds. To Err Is Human: Building a Safer Health System. Washington DC, USA: National Academy Press, 2000.

2. Currie M, Pybus DA, Torda TA. A prospective study of anaesthetic critical events: a report on a pilot study of 88 cases. Anaesth Intens Care 1989; 16: 98-100.

3. Sanborn KV, Castro J, Kuroda M, Thys DM. Detection of intraoperative incidents by electronic scanning of computerized anesthesia records. Anesthesiology 1996; 85: 977-987.

4. Runciman WB, Helps SC, Sexton EJ, Malpass A. A classification for incidents and accidents in the health-care system. J Qual Clin Pract 1998; 18: 199-211.

5. Webb RK, Currie M, Morgan CA, et al. The Australian Incident Monitoring Study: An Analysis of 2000 Incident Reports. Anaesth Intens Care 1993; 21: 520-528.

6. van Vuuren W, Shea CE, van der Schaaf TW. The Development of an Incident Analysis Tool for the Medical Field. Report EUT/BDK/85. Eindhoven, The Netherlands: Safety Management Group, Faculty of Technology Management, Eindhoven University of Technology, 1997.

7. De Keyser V, Nyssen AS. Les erreurs humaines en anesthésie. Le Travail Humain 1993; 56: 2-3, 233-241.
8. Johnson CW. Visualising the relationship between human error and organisational failure. In: Dixon M, ed. Proceedings of the 17th International Systems Safety Conference. Unionville, Virginia, USA: The Systems Safety Society, 1999: 101-110.

9. Caroll JM. Scenario-Based Design. New York, USA: Wiley \& Sons, 1995.

10. Cook R, Woods D, Miller C. A Tale of Two Stories: Contrasting Views of Patient Safety. Report from a Workshop on Assembling the Scientific Basis for Progress on Patient Safety. Chicago, USA: National Health Care Safety Council of the National Patient Safety Foundation at the AMA, 1998.

11. Busse DK, Johnson CW. Identification and analysis of incidents in complex, medical environments. Proceedings of the First Workshop on Human Error and Clinical Systems. Glasgow, UK: Glasgow Accident Analysis Group, Department of Computing Science, University of Glasgow, 1998.

12. Runciman WB. Iatrogenic Injury in Australia. Report of the Australian Patient Safety Foundation for the National Health Priorities and Quality Branch of the Department of Health and Aged Care of the Commonwealth. Government of Australia, 2000.

13. Nyssen AS, Hansez I, Baele P, De Keyser V, Lamy M. Occupational stress and burnout in anesthesia. $\mathrm{Br} J$ Anaesth 2003; 90: 333-337.

14. Lutsky I. Use of psychoactive substances among American anesthesiologists: a 30 year retrospective study. Can J Anaesth 1993; 40: 915-921.

15. Weeks AM, Buckland MR, Morgan EB, Myles PS. Chemical dependance in anaesthetic registrars in Australia and New Zealand. Anaesth Intens Care 1993; 21: 151-155. 